\title{
Percepción de la violencia de género de las mujeres Sordas mexicanas
}

\section{Perception of gender violence of Deaf Mexican women}

Benito Estrada Aranda

Universidad Autónoma de San Luis Potosí, San Luis Potosí, México benito.estrada@uaslp.mx https://orcid.org/0000-000I-78II-8525 (salud mental y sordera)

Silvia Romero Contreras

Universidad Autónoma de San Luis Potosí, San Luis Potosí, México silvia.romero@uaslp.mx

https://orcid.org/oooo-0002-7726-6195 (inclusión, comunicación)

Carmen Delgado Álvarez Universidad Pontificia de Salamanca, Salamanca, España cdelgado@upsa.es

https://orcid.org/0000-0002-6757-6317 (perspectiva de género, discapacidad)

Nicholas T. Kaufmann

Universidad Autónoma de San Luis Potosí, San Luis Potosí, México nicholas.kaufman@uaslp.mx https://orcid.org/o0oo-000I-6773-6459 (interseccionalidad)

ISSN-OI85-4259; e-ISSN: 2007-9176 DOI: http://dx.doi.org/IO.28928/ri/922022/aot2/estradaarandab/romerocontrerass

\section{Resumen}

Se cuenta con poca información sobre las experiencias de violencia de género de las mujeres Sordas en México. Este estudio cualitativo explora las percepciones acerca de tales experiencias en este grupo de población y ofrece una descripción general de los factores que intervienen en esa clase de conducta. Los resultados sugieren que las barreras de comunicación, la discriminación, la vulnerabilidad legal y el aislamiento social son prácticamente desconocidas en la sociedad mexicana, incluso dentro de las instituciones que trabajan para erradicar la violencia de género en la población en general. Además, este estudio identificó factores que directa o indirectamente llevan a las mujeres Sordas a experimentar violencia, incluido el abuso de sustancias, las relaciones interpersonales negativas y la sobreprotección dentro de los entornos familiar, educativo, laboral y social. Se discuten los resultados para promover el desarrollo de intervenciones dirigidas a las mujeres Sordas en México.

Palabras clave: discriminación, discapacidad, lengua de señas, grupos vulverables, inclusión.

\footnotetext{
Abstract

Little information is known about Deaf Women's experiences of gender violence in Mexico. This qualitative study explores perceptions of gender violence among this population group and offers a general description of factors that intervene in gender violence against Deaf women. Results suggest that communication barriers, discrimination, legal vulnerability and social isolation, are virtually unknown throughout Mexican society, even within institutions working to eradicate gender violence and in the general population. In addition, this study identified factors that directly or indirectly lead Deaf women to experience violence, including substance abuse, negative interpersonal relationships and overprotection within family, educational, work and social environments. Results are discussed to further the development of interventions targeting Deaf women in Mexico.
}

Key words: discrimination, disability, sign language, vulnerable groups, inclusion.

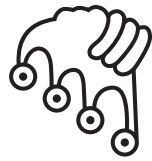

IZTAPALAPA

Agua sobre lajas 


\section{Introducción $^{1}$}

ara las mujeres con discapacidad que sufren violencia de género, el acceso a
los recursos sociales sigue siendo una asignatura pendiente, lo que facilita
la vulneración de sus derechos humanos (Estrada, 2008). En el caso de las mujeres Sordas, ${ }^{2}$ objeto de este estudio, el informe sobre su situación ante la violencia de género, elaborado por la Confederación Estatal de Personas Sordas en España (CNSE, 2009a), pone de relieve la doble discriminación e invisibilidad que padecen cuando son víctimas de malos tratos; como señala dicho informe, mientras las leyes garantizan su igualdad en el acceso a los recursos, la sociedad mantiene la discriminación al ofrecer recursos a los que no pueden acceder.

En países como México esta invisibilidad llega incluso a ignorarlas en las principales encuestas realizadas sobre violencia de género. Tal es el caso de la Encuesta Nacional sobre Violencia contra las Mujeres (Instituto Nacional de Salud Pública [INSP], 2003) y la Encuesta Nacional sobre la Dinámica de las Relaciones en los Hogares (endireh) (Instituto Nacional de Estadística y Geografía [INEGI], 2004; 2007; 2016), que excluyeron de sus muestras a mujeres con discapacidad, al contemplar como criterio de exclusión a aquellas que tuvieran alguna limitación para contestar por sí solas la encuesta. El estudio Mujeres y discapacidad en México realizado por el Instituto Nacional de las Mujeres (INM, 2002), es de los pocos en México que sí han incorporado en su muestra a mujeres Sordas.

1 Los autores expresan su agradecimiento a las alumnas de la Maestría en Psicología (Facultad de Psicología, uAslp) Nayeli Aréchiga Cervantes, María Alina Esparza Herrera, Mariana Castañeda Trejo y Maribel Candelaria Martínez, quienes colaboraron en el trabajo de campo y en la elaboración de la matriz de datos.

2 En este trabajo escribiremos mujer Sorda con "S" mayúscula para hacer referencia a las mujeres que tienen sordera profunda y que usan la lengua de señas para comunicarse. Mientras que las mujeres sordas con "s" minúscula serán aquellas que aun siendo sordas no usan la lengua de señas y no están inmersas en la cultura y comunidad sorda. 
Múltiples acciones y campañas sociales de organismos y asociaciones contra la violencia de género han planteado como objetivo "romper el silencio" que sufren las mujeres maltratadas. Estos programas y proyectos no alcanzan con igual eficiencia a grupos como las mujeres Sordas, por múltiples razones. En primer lugar, ellas no reciben la información transmitida por televisión o radio. Y en segundo, la información escrita publicada en prensa y las guías editadas por los organismos que promueven la equidad, por su alto contenido verbal, no está adaptada a las necesidades comunicativas de estas mujeres. Tales dificultades de acceso a la información son otro obstáculo para modificar las falsas creencias o "mitos" sobre la violencia de género, y producir el "cambio de mentalidad" necesario para "romper el silencio" sobre este problema. Las mujeres Sordas están más expuestas a las diversas formas de violencia ya que, en comparación con las demás, tienen mayor dependencia hacia su pareja, menor conciencia del abuso y menores índices de escolaridad (Ibarra y Muñoz, 20II).

Este trabajo es una primera aproximación al tema entre mujeres Sordas mexicanas. ${ }^{3}$ Se convocó a grupos de mujeres Sordas en los estados de Zacatecas, San Luis Potosí y en la Ciudad de México para conversar con ellas en forma individual y grupal sobre su percepción acerca de la violencia de género. A partir del análisis de sus testimonios, identificamos factores de riesgo y de protección respecto de la violencia de género en los ámbitos social, educativo, laboral y familiar.

\section{Antecedentes}

En México, según datos del INEGI (2010), hay cerca de medio millón de personas con discapacidad auditiva, de las cuales $54 \%$ son hombres y $46 \%$ mujeres. Ser persona Sorda significa pertenecer a una subcultura, ser miembro de un grupo que comparte una lengua común y que experimenta la discriminación a la que deben hacer frente los miembros de esa subcultura (Minguet Soto, 2000; Pino López, 2007). A la exclusión social hay que añadir además la dificultad que supone establecer redes dentro de la propia comunidad Sorda, debido a la diversidad de sus características respecto al problema que los identifica como parte de ese grupo. El informe de la Comisión de la Mujer de la CNSE (CNSE, 2009b) puso de manifiesto cómo en España

3 La realización de esta investigación ha sido posible gracias al financiamiento otorgado al primer autor por el Programa para el desarrollo profesional docente de la Secretaría de Educación Pública (Prodep-sep). 
esta diversidad debilita el establecimiento de redes entre las mujeres Sordas, por lo que "una mujer con discapacidad tiene mayor probabilidad de sufrir violencia de género que una mujer que no la tenga" (2009b: 40). Además, las dificultades de comunicación que sufren las mujeres Sordas, unidas a la falta de credibilidad que se otorga a quienes utilizan una lengua diferente y minoritaria y el propio desconocimiento de sus derechos las convierte en un grupo con un alto riesgo de sufrir algún tipo de maltrato dentro y fuera de la comunidad Sorda (CNSE, 2009a).

De la Rosa Prieto (2013), desde un análisis interseccional (Raj, 2002), afirma que la suma de la discriminación por género, más la propiciada por motivos de discapacidad y la generada por las distintas barreras sociales coloca a las mujeres con discapacidad en una situación de mayor vulnerabilidad con respecto a los hombres con discapacidad e incluso a las mujeres sin discapacidad. Diferentes estudios (Obinna et al., 2006; López González, 2007; Wojcik et al., 202I; Mason, 2010) muestran que las mujeres con discapacidad y, en especial, las mujeres Sordas, se mantienen en relaciones de malos tratos más tiempo debido a las barreras de comunicación y al menor número de servicios disponibles para resolver su situación. De acuerdo con Rems-Smario (2007: 17), "la violencia sexual y doméstica hacia la mujer Sorda continúa siendo invisible o incomprensible".

Existen diferentes tipos de violencia de género de la cual las mujeres en general suelen ser víctimas. Varios estudios han evidenciado la violencia física, verbal, sexual, emocional y/o económica que se ejerce contra mujeres (Casique y Castro, 2012), y particularmente contra mujeres Sordas (Watson, 20I4). En México, según la Ley general de acceso de las mujeres a una vida libre de violencia (Cámara de Diputados del H. Congreso de la Unión, 2015), la violencia de género se manifiesta en los ámbitos familiar, laboral, escolar, institucional y comunitario.

De acuerdo con las teorías feministas, la violencia de género contra las mujeres implica la supremacía de lo masculino sobre la mujer al generarle algún tipo de daño que se realice en contra de su voluntad (Frías, 2017). En efecto, existe suficiente evidencia que demuestra una correlación positiva entre las actitudes y creencias sexistas que reportan los hombres y la violencia que ejercen contra mujeres (McCarthy, Mehta y Haberland, 20I8), incluyendo mujeres Sordas (Ibarra y Muñoz, 20II).

No obstante, el ciclo de violencia (un fenómeno por el cual la victimización y la perpetuación aparecen inexorablemente vinculadas entre sí) también ha sido ampliamente documentado desde una perspectiva de desarrollo centrada en las distintas etapas de la vida (Frías, 2017; Wright et al., 2016). Por lo tanto, la explicación de la violencia de género descansa en la consideración de la interrelación de la violencia 
durante la infancia por la familia de origen con la que se da durante la vida adulta en ámbitos tales como relaciones de pareja o laborales.

Documentos como el elaborado por la Red Internacional de Mujeres con Discapacidad (International Network of Women with Disability, [INwwD], 20IO) y el estudio de Shum, Conde Rodríguez y Portillo Mallorca (2006) coinciden en que las personas con discapacidad son víctimas de maltrato y abuso en mayor medida que las personas sin discapacidad, y dentro de esta población, la violencia afecta a más a mujeres que a hombres. Dicho informe añade un elemento que agrava aún más la situación de este colectivo: las situaciones de maltrato tienden a ser más difícilmente reconocidas y atajables debido, entre otros motivos, al estigma asociado con la discapacidad y a los prejuicios sociales contra las mujeres con discapacidad en particular.

En México aún no existen datos oficiales sobre violencia de género y discapacidad, especialmente en el caso de las mujeres Sordas que, como vimos, suelen ser excluidas de las encuestas oficiales sobre violencia y dinámicas familiares En países como España la violencia de género contra mujeres con discapacidad es significativamente mayor que la ejercida en mujeres sin discapacidad: $18.8 \%$ en las primeras frente a I0.3\% en las segundas en 20II (Ministerio de Sanidad, Servicios Sociales e Igualdad [MSSSI], 20I2), en tanto que en 2015 el porcentaje de mujeres con discapacidad que sufrió violencia de género llegó a 31\% (MSssi, 2015).

\section{Metodología}

Dada la ausencia de estudios específicos sobre el tema en México, emprendimos un primer acercamiento, con base en una metodología cualitativa, con el objetivo de conocer la percepción que tienen mujeres Sordas en México sobre la violencia de género recopilando sus opiniones y experiencias sobre el tema, lo que nos permitiría otorgar el principal protagonismo a las implicadas. En el diseño seguimos algunos de los lineamientos del estudio realizado por el CNSE (2009a) con mujeres Sordas en España.

Se trabajó en tres ciudades mexicanas en las que había facilidades de acceso a la población meta: San Luis Potosí, Zacatecas y la Ciudad de México. En San Luis Potosí se contactó a la Asociación de Sordos de San Luis Potosí y la Fundación Abres mi Luz. En Zacatecas se estableció contacto con una intérprete de Lengua de Señas Mexicana, quien se encargó de convocar y citar a las participantes. En la Ciudad de México se realizaron gestiones con el Instituto Pedagógico para Pro- 
blemas del Lenguaje (IPPLIAP) para que convocara a un grupo de mujeres sordas vinculadas al Instituto.

Se utilizaron dos técnicas cualitativas: grupos focales o de discusión (Bloor et al., 200I; Morgan, 1997) y entrevistas individuales (CNSE, 2009a). Se realizaron tres grupos de discusión, uno en cada ciudad, integrados por mujeres Sordas y ocho entrevistas a mujeres que fueron víctimas o testigos de la violencia de género en San Luis Potosí.

Los grupos focales se llevaron a cabo por el primer autor de este estudio. En ellos se abordaron temáticas relacionadas con la percepción que tenían las participantes sobre la violencia desde su significado hasta experiencias cercanas de situaciónes de violencia, sobre cómo viven la violencia las mujeres Sordas, qué hacen cuándo la sufren, a quién recurren para pedir ayuda, qué formas de violencia conocen, o qué diferencias perciben entre la violencia hacia mujeres sordas y hacia mujeres oyentes.

Para las entrevistas se siguió el protocolo de estudio de CNSE (2009a) que aborda cuestiones de información sociodemográfica, contexto personal y familiar y experiencias de violencia de género. La duración promedio de los grupos focales fue de 62 minutos y las entrevistas de 36 minutos, en total se obtuvieron 7.5 horas de material cuya transcripción arrojó ioo páginas. Durante el trabajo de campo se contó con el apoyo de tres mujeres intérpretes de LSM con más de io años de experiencia y reconocidas por la comunidad sorda como intérpretes de calidad ${ }^{4} y$ se realizó registro en video, previo consentimiento informado de las participantes. Para la traducción y transcripción de las entrevistas se contó con el apoyo de una mujer con hipoacusia que conoce la LSM.

Las participantes recibieron una carta de consentimiento informado que la intérprete les tradujo a la LSM en la que se aseguraba la confidencialidad absoluta de los datos generados por su participación, omitiendo su nombre en las publicaciones derivadas del proyecto.

La selección de las intérpretes se basó en recomendaciones de la misma comunidad Sorda, dado que hay muy pocos profesionales en este ramo y no existe un padrón de intérpretes oficial. En 2009 el Consejo Nacional de Normalización y Certificación de Competencias Laborales (CONOCER) emitió la norma para el servicio profesional de interpretación de LSM al español y viceversa (NUIPDooi.oI, Diario Oficial de la Federación [DOF]) 2009) cuya observancia es limitada y no ha sido actualizada. Además, el conoCER, órgano responsable de la certificación de intérpretes de LSM, ha dejado de cumplir con ese proceso aduciendo falta de interés (Godoy, 2015). Según el portal de noticias SIPSE.com (I-octubre-20I6) en México hay apenas 40 intérpretes de señas certificados de los cuales la cuarta parte está en la Ciudad de México. 


\section{Participantes}

Se entrevistó individualmente a ocho mujeres de San Luis Potosí y en los grupos focales participaron un total 28 mujeres. Como se observa en el cuadro I, el rango de edad de las participantes fue similar para SLP y Zacatecas (I9 a 48 años) en tanto que en Ciudad de México el rango se extiende hasta los 7I años. Un poco más de la mitad de las participantes son solteras y un tercio son casadas. Mientras que las participantes de SLP y Zacatecas cuentan con años de escolaridad promedio de entre 7 y II años, para las de Ciudad de México el promedio es de I3 años. En cuanto a ocupación, predominan la dedicación al hogar, ser estudiante y el autoempleo. Respecto de sus relaciones, un poco más de la mitad de las participantes declaró tener una pareja sorda y todos los hijos de las participantes son oyentes.

Las características generales de vida de las mujeres Sordas que participaron en este estudio pueden analizarse en función de las diversas capas de vulnerabilidad que implican ante la violencia de género. Identificamos que además de ser mujeres y sordas, las participantes presentan otras varias características o capas de vulnerabilidad (véase cuadro I) tales como: ser madre soltera, tener hijos oyentes, tener una pareja oyente, contar solo con escolaridad básica, no contar con un ingreso personal por dedicarse al hogar, tener trabajos de medio tiempo o ser autoempleada con lo fluctuante que esto puede resultar, y vivir en provincia, en oposición a vivir en la capital del país, donde hay mayores desventajas en cuanto a accesibilidad, inclusión y oportunidades. Al identificar estas características intentamos dar cuenta, tal y como lo plantea con perspectiva interseccional De la Rosa (2013), acerca de la conformación de procesos de discriminación multinivel por la suma de estas capas de vulnerabilidad.

\section{Cuadro I.}

Características sociodemográficas de las participantes

\begin{tabular}{|c|c|c|c|c|c|}
\hline & $\begin{array}{c}\text { SLP } \\
\text { Entrevistas } \\
n=8\end{array}$ & $\begin{array}{c}\text { SLP } \\
\text { GPOFI } \\
n=13\end{array}$ & $\begin{array}{c}\text { ZAC } \\
\text { GPOF2 } \\
n=6\end{array}$ & $\begin{array}{c}\text { CDMX } \\
\text { GPOF3 } \\
n=9\end{array}$ & $\begin{array}{l}\text { Totales } \\
N=36\end{array}$ \\
\hline Rango de edad & $2 I-48$ & $19-46$ & $28-45$ & $20-71$ & $19-71$ \\
\hline Edad media & 32 & 25.4 & 35 & 40.2 & -- \\
\hline \multicolumn{6}{|l|}{ Estado civil: } \\
\hline Casada & 5 & I & 4 & 3 & 13 \\
\hline Soltera & 3 & $\mathrm{I} 2$ & 2 & 2 & 19 \\
\hline Separada & & & & 2 & 2 \\
\hline
\end{tabular}




\begin{tabular}{|c|c|c|c|c|c|}
\hline & $\begin{array}{c}\text { SLP } \\
\text { Entrevistas } \\
n=8\end{array}$ & $\begin{array}{c}\text { SLP } \\
\text { GPOFI } \\
n=13\end{array}$ & $\begin{array}{c}\text { ZAC } \\
\text { GPOF2 } \\
n=6\end{array}$ & $\begin{array}{c}\text { CDMX } \\
\text { GPOF3 } \\
n=9\end{array}$ & $\begin{array}{l}\text { Totales } \\
N=36\end{array}$ \\
\hline Divorciada & & & & I & I \\
\hline Unión libre & & & & I & I \\
\hline \multicolumn{6}{|l|}{ Escolaridad: } \\
\hline Sin estudios & 2 & 2 & & I & 5 \\
\hline Primaria & 2 & & 4 & & 6 \\
\hline Secundaria & & 6 & & 3 & 9 \\
\hline Preparatoria & I & & I & 3 & 5 \\
\hline Carrera técnica & I & 2 & & & 3 \\
\hline Licenciatura & & I & I & 2 & 4 \\
\hline Desconocido & 2 & 2 & & & 4 \\
\hline $\begin{array}{l}\text { Educación } \\
\text { predominante moda }\end{array}$ & $\begin{array}{c}\text { Sin estudios } \\
\text { Primaria }\end{array}$ & Secundaria & Primaria & $\begin{array}{l}\text { Secundaria } \\
\text { Preparatoria }\end{array}$ & - \\
\hline Media en años & 7 & II & 9.5 & I3 & - \\
\hline \multicolumn{6}{|l|}{ Ocupación: } \\
\hline Hogar & 5 & I & 2 & 3 & II \\
\hline Auto-empleada & I & 2 & 3 & 2 & 8 \\
\hline $\begin{array}{l}\text { Empleada de medio } \\
\text { tiempo }\end{array}$ & 2 & & I & & 3 \\
\hline Estudiante & & IO & & & IO \\
\hline Profesionista & & & & 4 & 4 \\
\hline Pareja sorda & 4 & 8 & 4 & 3 & 19 \\
\hline Hijo/a (s) oyentes & 2 & I & I & 6 & IO \\
\hline
\end{tabular}

\section{Enfoque analítico}

La construcción de las categorías se realizó mediante la revisión iterativa de los datos, siguiendo la metodología de la teoría fundamentada (Strauss y Corbin, 2002) de codificación abierta, axial y selectiva. Inicialmente, la discusión de los datos tuvo 
lugar en una comunidad interpretativa (CI) integrada por cuatro maestrantes ${ }^{5} y$ dos asesores, ${ }^{6}$ quienes asistían a las sesiones presenciales y realizaban revisiones de memorandos analíticos.

Para la codificación, cada integrante de la ci categorizó los datos de forma inductiva. Este primer análisis se trianguló entre los participantes con la finalidad de verificar la concordancia. La categorización implicó la búsqueda de patrones recurrentes en el discurso a través de la lectura repetida los textos (codificación abierta); después, a cada argumento se le asignó una etiqueta general, categoría madre; posteriormente se realizaron subdivisiones de las categorías (codificación axial). Al final de este proceso quedaron nueve categorías madre con sus categorías hijas (más específicas).

Una vez definido el sistema de categorías (en dos niveles: madres e hijas) se realizó la codificación selectiva a partir de la categoría de violencia, que constituye el tema central de este estudio, utilizando el modelo de diagramación. Se realizaron varios diagramas de las relaciones entre la categoría central y las demás categorías para explicar las relaciones y los procesos que la explican y le dan origen. La construcción de los diagramas fue guiada por la pregunta: ¿Cómo se presenta la violencia de género en mujeres Sordas?

En este proceso, que es similar al rastreo de una senda propuesto por Strauss y Corbin (2002), identificamos varios circuitos, que luego corroboramos en los datos, identificando su presencia en una o más participantes. Los circuitos más productivos, es decir, aquellos que se corroboraron en un mayor número de casos, se consideraron circuitos explicativos del fenómeno de violencia en las mujeres Sordas. En la sección de resultados se presentan las categorías y los circuitos identificados.

En total se realizaron cuatro diagramas. El primero fue interpretativo y general. El segundo, también interpretativo, se dividió en factores protectores y de riesgo. El tercer diagrama resumió los diagramas anteriores. El último diagrama, que es el que se presenta en los resultados de este trabajo, tomó como principal criterio la productividad de las categorías y su interpretación.

5 El trabajo de la comunidad interpretativa fue fundamental para el desarrollo del análisis; no obstante, esta comunidad no pudo continuar, por lo que las conclusiones de esta se revisaron por los autores $y$, en muchos casos se hicieron cambios de fondo. Los autores agradecen a Nayeli Aréchiga Cervantes, María Alina Esparza Herrera, Mariana Castaneda Trejo y Maribel Candelaria Martínez su contribución en el proceso analítico.

6 El primer autor y la segunda autora coordinaron y asesoraron los trabajos de la comunidad interpretativa como parte de un proceso formativo. 
La revisión de categorías y de la diagramación fue iterativo, es decir, los productos fueron cambiando a partir de las revisiones de la comunidad, tomando como criterio principal la productividad de las categorías y la revisión reiterativa de los datos. Tanto durante la elaboración de las categorías como durante el proceso de diagramación, cuando se encontraron discrepancias entre los miembros de la comunidad interpretativa se realizaron revisiones y modificaciones, hasta que todos estuviesen de acuerdo con los productos generados. En total la comunidad interpretativa se reunió aproximadamente is veces para la revisión exhaustiva de los datos. A lo largo del proceso, los asesores ofrecieron apoyo para ir afinando el análisis de datos, aportar nuevas perspectivas, enriquecer el análisis y generar debates y nuevas hipótesis entre los maestrantes.

Después de la codificación inicial realizada por la ci y para darle mayor solidez a los datos, se realizó una codificación inter-jueces para determinar la confiabilidad de las categorías. Se eligieron aleatoriamente dos textos: una entrevista de seis páginas de San Luis Potosí (texto I) y el grupo focal de Zacatecas de I5 páginas (texto 2), lo que representa $20 \%$ del material transcrito. Se entrenó a II jueces, maestrantes en psicología de la Universidad Autónoma de San Luis Potosí con experiencia en análisis cualitativo, en el manejo de las categorías y el procedimiento de codificación, y se les pidió que codificaran segmentos previamente seleccionados y que cuando no estuvieran seguros del código dejaran el segmento sin codificar. Cada juez codificó solo un texto.

Se analizaron las coincidencias entre jueces de cada segmento y se obtuvo un porcentaje de coincidencia con base en el número de jueces que evaluó cada segmento. Así, si había cuatro coincidencias y seis jueces se registró un porcentaje de coincidencia de $67 \%$ para ese segmento. En el cuadro 2 se presenta el resumen de análisis por cada texto.

\section{Cuadro 2.}

Resultados del análisis inter-jueces de las categorías madre e hija

\begin{tabular}{lccccccc}
\hline & No. de & \multicolumn{3}{c}{ No. de jueces } & \multicolumn{4}{c}{ Coincidencia } \\
\cline { 3 - 8 } Texto & $\begin{array}{c}\text { segmen- } \\
\text { tos }\end{array}$ & Total & $\begin{array}{c}\text { Evaluó } \\
\leq 80 \% \text { de los } \\
\text { segmentos }\end{array}$ & $100 \%$ & $80 \%$ & $67-70 \%$ & $50 \%$ \\
\hline I. Entrevista SLP & 29 & 6 & 6 & $31 \%$ & $62 \%$ & $79 \%$ & $100 \%$ \\
\hline 2. Grupo enfoque zAC & 34 & 5 & 3 & $23 \%$ & $29 \%$ & $53 \%$ & $94 \%$ \\
\hline
\end{tabular}


El análisis de confiabilidad muestra que las coincidencias unánimes, es decir, de todos los jueces, son de $30 \%$ o menos; sin embargo, las coincidencias parciales se incrementan considerablemente. Cabe señalar que el número de jueces fue de cinco a seis - alto - para cada muestra, por lo que obtener coincidencias unánimes en una clasificación cualitativa resulta poco probable. Es importante señalar que en este registro solo se tomaron en cuenta coincidencias exactas de la codificación, y no se ponderaron las parciales (misma categoría madre con diferente categoría hija) o similares. Por otra parte, los datos del texto I, derivados de una entrevista individual, fueron mucho más claros y precisos para los jueces, en tanto que los del texto 2, proveniente de un grupo focal, resultaron menos claros para su codificación.

\section{Resultados}

En esta sección se presentan las categorías desarrolladas y empleadas en el análisis, con ejemplos extraídos de los datos recabados (cuadro 3). Posteriormente, se presentan, describen y ejemplifican las relaciones entre las categorías identificadas en los textos (cuadro 4), a las que hemos denominado circuitos. Por último, se integran los hallazgos en un diagrama final.

Las categorías que aparecen en el cuadro 3 dan cuenta de tipos de violencia de género, factores protectores, factores de riesgo, así como la percepción de algunas de las participantes sobre sí mismas y su papel en la sociedad. En los párrafos siguientes hacemos algunas consideraciones generales respecto de las categorías identificadas. $\mathrm{Al}$ referirnos a ellas las indicamos en cursivas.

Las participantes, al manifestar su percepción acerca de la violencia de género, hicieron referencia a distintos tipos, física, psicológica, sexual y económica. Además de la violencia, como tal, también hicieron mención de actos de discriminación y sobreprotección por la discapacidad que, desde cierto punto de vista, pueden considerarse una forma de violencia; sin embargo, éstas se separaron en otra categoría para facilitar el análisis y la comprensión del fenómeno.

En las conversaciones emergieron factores de riesgo hacia la violencia como el abuso de sustancias en la familia, las relaciones familiares negativas y distantes y la falta de estrategias de comunicación. También surgieron algunos factores protectores, entre los que destacan las relaciones familiares cercanas y positivas, el apoyo social por parte de amigos, familiares e instituciones y el desarrollo de estrategias de comunicación diversas y semejantes a las de la comunidad oyente. Algunas participantes hicieron comentarios sobre sus expectativas a futuro, las cuales, por lo general fueron positivas. 
Las participantes también se refirieron a su autopercepción y a cómo entienden que la sociedad percibe a las personas con sordera (sociopercepción). En términos generales, los testimonios muestran que la percepción propia y de otros las pone en desventaja en relación con la población oyente.

\section{Cuadro 3.}

Categorias madre e bija con ejemplos ${ }^{7}$

\begin{tabular}{lll}
\hline Categorías madre/ hijas & Ejemplo \\
\hline 1. & Violencia de Género & \\
\hline 1.1. & Violencia física. & $\begin{array}{l}\text { Un poco de violencia, jaloneos sí, pero ella le } \\
\text { pone sus límites y no se deja... (M, SLP, 29).HR }\end{array}$ \\
\hline $\begin{array}{l}\text { 1.2. } \\
\begin{array}{l}\text { Violencia psicológica. Refiere } \\
\text { insultos, humillaciones, ame- } \\
\text { nazas, chantajes, abandono, } \\
\text { exclusión. }\end{array}\end{array}$ & $\begin{array}{l}\text { No me querían mandar a la escuela porque } \\
\text { creían que no iba a aprender (H, SLP, 45). }\end{array}$ \\
\hline $\begin{array}{l}\text { Violencia sexual. Refiere } \\
\text { coacción con el objeto de una } \\
\text { determinada conducta sexual, } \\
\text { insinuaciones sexuales. }\end{array}$ & $\begin{array}{l}\text { Ella no quería tener relaciones pero el novio la } \\
\text { forzó... (M, sLP, 29) HR. }\end{array}$ \\
$\begin{array}{l}\text { Violencia económica. Refiere } \\
\text { coacción basado en el apoyo } \\
\text { económico condicionado. }\end{array}$ & $\begin{array}{l}\text { No me dice nada, me da poco dinero, a veces } \\
\text { tenemos conflictos, yo le digo por qué te vas } \\
\text { a vender, o sea te vas tanto tiempo a vender y } \\
\text { sigues trayendo lo mismo para comer, ay si yo } \\
\text { se que te va bien cuando cobras, (J, sLP, 48). } \\
\text { [refiriéndose a su pareja] }\end{array}$ \\
\hline
\end{tabular}

\section{Discriminación/ sobreprotección por discapacidad.}

2.1. Discriminación laboral. En el trabajo muchos problemas, discriminación por ser mujer sorda, se peleaba con sus compañeros, por los problemas con sus compañeros renuncio, porque no sabía que tenía derechos... $(\mathrm{M}, \mathrm{SLP}, 29) \mathrm{HR}$

7 En los ejemplos, se emplean las siguientes abreviaturas para indicar la fuente del testimonio $:$ GF = Grupo focal; SLP = San Luis Potosí; $\mathrm{ZAC}=$ Zacatecas; $\mathrm{CM}=$ Ciudad de México. Para los casos de entrevista se proporciona, además de la ciudad, la inicial con la que se identifica a la participante y su edad. Al final de la identificación de la informante se indica HR cuando se trata de habla reportada, es decir, cuando la intérprete no dio el testimonio de manera literal, sino como reporte de lo dicho. 


\begin{tabular}{ll}
\hline Categorias madre/ hijas & Ejemplo \\
\hline 2.2. Discriminación familiar. & Si se ha sentido discriminada con la familia por \\
& los problemas de comunicación y de que luego \\
& pide que le expliquen una palabra o le digan algo \\
& y le dicen iespérate!... (M, sLP, 29) HR. \\
\hline
\end{tabular}

2.3. Discriminación social. La gente te humilla te dice ignorante, que estás mal, o sea aquí es eso (C, SLP, 28).

2.4. Discriminación en la escuela. Los maestros no me tenían paciencia y me salí (H, SLP, 45).

2.5. Sobreprotección familiar, por No me gusta que antes mi papá me sobreproteprejuicio negativo asociado a gía más que al resto de mis hermanos $(\mathrm{H}, \mathrm{SLP}$, la sordera. 45).

3. Abuso de sustancias en la A veces me gustaría quedarme en mi casa tranfamilia. quila pero mi papá todo el tiempo toma (GF, SLP).

4. Relaciones familiares.

4.1. Conflictos repetitivos entre Ella cuando estaba niña siempre los vio peleánlos padres. dose, siempre, ella recuerda (GF, ZAC) HR

4.2. Relación cercana, positiva Su mamá vive en el mismo lugar es muy buena, con la madre. a ella le gusta mucho pasar tiempo con su mamá. (GF, ZAC) HR

4.3. Relación distante, negativa La relación era muy conflictiva, había rivalidad con la madre. entre ellas. Cuando la regañaba le pegaba, pero más a ella que a su hermano... (M,SLP,29) HR

4.4. Relación cercana, positiva con La relación con su papá siempre fue mejor, conel padre. sidera que gracias a él aprendió a leer y escribir y que tiene un conocimiento más amplio gracias a su papá (M,SLP,32) HR

4.5. Relación distante, negativa Pues en lengua de señas no hay, no hay comunicon el padre. cación, si no sabe pues no hay, no hay (J,SLP,48) [refiriéndose a que su padre no sabe lengua de señas].

4.6. Relación cercana, positiva con Los hermanos si la querían, la cuidaban, tenían los hermanos. problemas pero si se ayudaban entre ellos... (M,SLP,29). HR 


\begin{tabular}{cl}
\hline Categorías madre/ hijas & Ejemplo \\
\hline $4.7 . \quad$ Relación distante, negativa & Muy poco, en realidad muy poco, con ellos tres si \\
con los hermanos. & me llevaba más, entonces yo les enseñaba señas \\
& y ellos me interpretaban y ellos hablaban y le \\
& interpretaban como interpretes ¿̨no? nada más. \\
& $(\mathrm{J}, \mathrm{SLP}, 48)$. \\
\hline
\end{tabular}

4.8. Relación cercana, positiva con Mi hijo el mayor es el que me da y me sorprende loshij@s. ¿no? yo lo quiero mucho, yo lo amo, porque él me trata muy bien, él es bueno, yo le agradezco, y gracias a él, para comer bien y cada mes cada mes cada mes (J,SLP,48).

4.9. Relación cercana, positiva Me atiende, siempre está al pendiente, me ayuda con la pareja. a lo que sea, lo que necesite siempre me ayuda $(\mathrm{A}, \mathrm{SLP}, 24)$ [ haciendo referencia a la pareja].

4.10. Relación distante, negativa con la pareja . El último novio que tuvo fue oyente, terminó la relación por que se sentía discriminada y los problemas de comunicación (M,SLP,32). HR

\section{Apoyo social recibido}

5.1. Amigos. Sus amigas le han ayudado y le han explicado que pos no pasa nada, que debe de hacerse fuerte, que tenga confianza, que nada se sentimentalismos, que se haga fuerte, y ya que esté tranquila (GF, ZAC) HR

5.2. Familiares La relación con su papá siempre fue mejor, considera que gracias a él aprendió a leer y escribir y que tiene un conocimiento más amplio gracias a su papá... (M, SLP, 32) HR.

5.3. Instituciones. Estuve yendo a una asociación de sordos (H,SLP,45).

6. Estrategias de comunicación empleadas.

6.1. Lengua de señas. Me comunico más con lengua de señas (H, SLP, 45).

6.2. Mímica o teatro. Ella [persona de su familia] trataba como actuando la situación, explicarme que era lo que pasaba, usaba mucho la cosa teatrera para explicarme (H, SLP, 32).

6.3. Lectura de labios. Pero también uso lectura labial (H, SLP, 45). 


\begin{tabular}{|c|c|c|}
\hline \multicolumn{2}{|c|}{ Categorias madre/ hijas } & \multirow{2}{*}{$\begin{array}{l}\text { Ejemplo } \\
\ldots \text { y algo de comunicación oral (H, SLP, 45). }\end{array}$} \\
\hline 6.4 & Lenguaje oral vía oralización. & \\
\hline 6.5 & Lectura. & $\begin{array}{l}\text { Su comprensión de lectura y escritura es muy } \\
\text { buena porque su papá le enseñó (M, SLP, } 32) . H R\end{array}$ \\
\hline 6.6 & Escritura. & $\begin{array}{l}\text { Su comprensión de lectura y escritura es muy } \\
\text { buena porque su papá le enseño }(M, S L P, 32) . H R\end{array}$ \\
\hline 7. & Autopercepción. & $\begin{array}{l}\text { Yo creo que los sordos y los oyentes no creo } \\
\text { que seamos exactamente iguales, yo creo que } \\
\text { los sordos tienen un poco, no sé cómo decirlo } \\
\text { no sé, más como inocencia, los oyentes saben } \\
\text { muchísimas cosas de lo que escuchan pero el } \\
\text { sordo no, no, no tiene una manera de cómo te } \\
\text { entre todo eso como te ayuden (GF, SLP). }\end{array}$ \\
\hline 8. & Socio-percepción & \\
\hline 8.1 . & $\begin{array}{l}\text { Socio-Percepción de la } \\
\text { comunidad sorda. }\end{array}$ & $\begin{array}{l}\text { No, los sordos viven más tiempo en casa de sus } \\
\text { papás porque no tienen dinero, entonces no se } \\
\text { quieren casar pronto. Sólo buscan juntarse, no } \\
\text { tienen dinero y quieren que vivamos en casa de } \\
\text { sus papas. Y los oyentes sí tienen dinero y se } \\
\text { quieren casar pronto }(\mathrm{H}, \mathrm{SLP}, 45) \text {. }\end{array}$ \\
\hline 8.2 & $\begin{array}{l}\text { Socio-Percepción de la } \\
\text { comunidad oyente. }\end{array}$ & $\begin{array}{l}\text { Los oyentes tienen un vocabulario más amplio, } \\
\text { saben mucho (H, SLP, } 45\end{array}$ \\
\hline 8.3. & $\begin{array}{l}\text { Socio-Percepción de roles de } \\
\text { género. Normas sociales y } \\
\text { comportamentales percibidas } \\
\text { como propias del género }\end{array}$ & $\begin{array}{l}\text { ¡Ay mira! yo soy hombre y sé lenguaje de señas } \\
\text { muy bien y tú eres mujer y no sabes bien]. Tiene } \\
\text { que ver cómo esta lucha de poderes de yo soy } \\
\text { mejor, yo si sé, tú no sabes (GF, CM)[recreando } \\
\text { un diálogo dicho por un varón] }\end{array}$ \\
\hline 9. & Expectativas a futuro. & $\begin{array}{l}\text { Le gustaría trabajar en lenguaje de señas para } \\
\text { niños, como maestra de lenguaje de señas (M, } \\
\text { SLP, 32). HR }\end{array}$ \\
\hline
\end{tabular}

Derivado del análisis de cómo se relacionan las categorías se identificaron siete circuitos que explican los factores de riesgo asociados con la violencia, así como los factores protectores. En el cuadro 4 se presentan los circuitos y el código del informante en el cual se encontró el circuito. Como se puede observar, hubo algunos circuitos más productivos, es decir, que se presentaron hasta en seis informantes o grupos, en tanto que otros fueron poco productivos, en solo dos informantes o grupos. 
Cuadro 4. Productividad de los circuitos.

\begin{tabular}{|c|c|c|c|c|}
\hline TIPO DE FACTOR & CIRCUITO & INFORMAN & & \\
\hline \multirow{7}{*}{ DE RIESGO } & \multirow{2}{*}{$\begin{array}{l}\text { Discriminación / sobrepro- } \\
\text { tección - Violencia }\end{array}$} & GF, ZAC & M, SLP, 29 & $\mathrm{~J}, \mathrm{SLP}, 48$ \\
\hline & & M, SLP, $2 I$ & GF, SLP & $\mathrm{GF}, \mathrm{CM}$ \\
\hline & \multirow{2}{*}{$\begin{array}{l}\text { Relación familiar negati- } \\
\text { va-discriminación-violencia }\end{array}$} & GF, SLP & M, SLP, 29 & $\mathrm{~J}, \mathrm{SLP}, 48$ \\
\hline & & A, SLP, 24 & $\mathrm{M}, \mathrm{SLP}, 2 \mathrm{I}$ & M, SLP, 32 \\
\hline & $\begin{array}{l}\text { Discriminación - } \\
\text { Autopercepción }\end{array}$ & M, SLP, 29 & $\mathrm{M}, \mathrm{SLP}, 32$ & J, SLP, 48 \\
\hline & $\begin{array}{l}\text { Socio percepción - } \\
\text { Discriminación }\end{array}$ & A, SLP, 24 & $\mathrm{GF}, \mathrm{CM}$ & \\
\hline & $\begin{array}{l}\text { Abuso de sustancias - } \\
\text { violencia }\end{array}$ & GF, SLP & GF, ZAC & \\
\hline \multirow{3}{*}{ PROTECTOR } & \multirow{2}{*}{$\begin{array}{l}\text { Apoyo social- Estrategias de } \\
\text { comunicación }\end{array}$} & $\mathrm{H}, \mathrm{SLP}, 32$ & GF, ZAC & \\
\hline & & GF, SLP & M, SLP, 32 & \\
\hline & $\begin{array}{l}\text { Estrategias de comunicación } \\
\text {-Autopercepción }\end{array}$ & GF, SLP & GF, DF & \\
\hline
\end{tabular}

\section{Descripción de los circuitos}

\section{Discriminación/sobreprotección-violencia}

En los relatos de las mujeres Sordas la discriminación y la sobreprotección aparecen de manera conjunta, particularmente dentro de la dinámica familiar. Así, en un intento por no exponer a las participantes a peligros o a situaciones inconvenientes o que suponen que no pueden enfrentar, les restringen sus libertades y solo les permiten funcionar en ciertos entornos y para realizar ciertas actividades, con lo que las sobreprotegen y al mismo tiempo las discriminan, pues el trato que les dan es distinto al que reciben otros miembros no sordos o no mujeres de la familia. Además, la sociedad en general ejerce discriminación hacia las mujeres Sordas especialmente por su condición de sordera y su dificultad para comunicarse. Tanto la discriminación como la sobreprotección son factores de riesgo que derivan en violencia, como se muestra en los siguientes ejemplos. 
J, SLP, 48, comentó respecto de su padre lo siguiente: “Me encerraba, yo no podía salir, mi papá decía que era porque tenía miedo a que me pasara algo...". También mencionó que no la dejaban ir a la escuela:

no tú no vas a ir a la escuela; y yo ni siquiera con señas o algo que me explicara por qué yo no podía ir a la escuela, y luego me encontraban y me decían: ¿tú por qué no estás bordando? ¿por qué no estás cosiendo? Hubo una discusión, una clase de discusión y estaban hablando entre los dos [madre y padre], estaban discutiendo, y entonces me empezaron a decir: no, es que ¡tú eres burra! ¡Tú eres burra!

En este ejemplo puede observarse que el temor del padre de que le sucediera algo a su hija lo llevó a actuar de forma sobreprotectora, con violencia psicológica, encerrándola en casa, impidiéndole el contacto social con otras personas $y$, por consecuencia, el desarrollo de habilidades sociales.

En el grupo focal realizado en San Luis Potosí (GF, SLP) surgió el siguiente relato:

trataba de hablar con los oyentes, pero de todas maneras, aunque lo intentaba no podía encajar y se burlaban de mí, por ejemplo, mi hermana mayor se burlaba mucho; todos me decían groserías cuando no había alguien que me defendiera o que me cuidara era pues todo un sufrimiento ¿no?, y si no era estar encerrada en la casa ahí noche y día.

También en el mismo grupo focal (GF, SLP), otra participante relata:

en la escuela, hay maestro muy grosero que no le gustaba la lengua de señas y nos daba de manazos y nos decía, ;habla, habla! - nos pegaba en las manos, no nos dejaba hacer señas, nos jalaba de las orejas, a todas, a todas las compañeras ...; mi mamá mejor me sacó de esa escuela y me mandó a una escuela de oyentes porque cómo iba a estar conviviendo con esas personas [compañeras Sordas que signaban].

En estos casos, observamos que las participantes eran discriminadas cuando trataban de comunicarse: una por usar lenguaje oral, otra por usar LSM. Esta discriminación causó que fueran víctimas de violencia psicológica y física. Estos dos relatos evidencian la complejidad de las personas sordas, quienes frecuentemente han carecido de formas de comunicación efectiva o aceptable en su medio más inmediato: casa y escuela. 


\section{Relación familiar distante o negativa-discriminación-violencia}

La categoría de relación familiar fue subdividida en relación familiar distante o negativa y relación familiar cercana. En este circuito se presenta una asociación entre relación familiar distante o negativa y la discriminación y/o la violencia. En los discursos es frecuente encontrar que cuando las participantes percibieron este tipo de relación con los miembros de su familia también reportan situaciones de discriminación y de franca violencia, como se muestra en los siguientes ejemplos.

Algunos de los datos de relaciones familiares distantes o negativas que emergieron en los discursos de las participantes se vinculan con procesos de discriminación, particularmente en razón de su poca capacidad de comunicación con los miembros oyentes de la familia, como se muestra en los siguientes ejemplos. Cabe señalar que la abrumadora mayoría de las personas con sordera nacen en hogares oyentes (Mitchell y Karchmer, 2004; Muñoz, 2007).

M, SLP, 32 comentó a través de la intérprete:

Su papá siempre le pegaba por traviesa, pero solo por traviesa, pero nunca por otra cosa...la mamá únicamente regaños, regaños ... dice que es discriminada, la única [sorda] en su familia. Sí se sentía discriminada porque es la única en su familia y su familia en sí, entonces el problema de comunicación y cómo sentirse parte de una familia oyente, se sentía triste... por ser mujer sorda, su principal problema, la depresión que ella siente.

Por otra parte, como se aprecia en los ejemplos siguientes, las relaciones con distintos miembros de la familia generan desde violencia psicológica, como es el control, hasta violencia física

M, SLP, 2I expuso:

pero con mi hermano, chocamos y chocamos y chocamos muchísimo porque él trata de decirme cómo me debo de comportar, cómo debo de ser, quiere enseñarme a ser como supuestamente muy madura, no me deja, me controla, así siempre es mi hermano, yo sé que es mi hermano y tenemos choques frecuentes.

M, SLP, 29, relata la relación con su madre: "la relación era muy conflictiva, había rivalidad entre nosotras. Cuando me regañaba me pegaba, pero más a mí que a mi hermano". 


\section{Apoyo social-estrategias de comunicación}

Los datos indican que el desarrollo de estrategias de comunicación se ve favorecido por el apoyo, ya sea al interior de la familia o en otros círculos sociales o institucionales. En el primer ejemplo, la participante hace referencia a la convivencia en la comunidad Sorda como central en su conocimiento de la LSM. En el siguiente, explica el papel que tuvo un integrante de su familia en el desarrollo de sus habilidades comunicativas.

Grupo focal de Zacatecas (GF, ZAC): "cuando empecé a conocer a los sordos y sus esposas empecé a tener un trato mejor, y empecé también a aprender más el lenguaje [de señas], se nos abre la mente y me puedo expresar más porque empiezo a aprender más el lenguaje".

M, SLP, 32, comenta, vía el relato de la intérprete: "solo estudió la primaria su comprensión de lectura y escritura son muy buenas porque su papá [quien es maestro de secundaria] le enseñó, considera que gracias a él aprendió a leer y escribir y que tiene un conocimiento más amplio"

\section{Discriminación-autopercepción}

Las situaciones de discriminación, al paso del tiempo, pueden moldear el sentido de autopercepción de tal forma que la persona sorda se perciba inferior. El discurso de la siguiente participante ilustra lo anterior al señalar que la constante discriminación por parte de su esposo le provoca una sensación de inferioridad.

J, SLP, 48 comenta: "yo le he dicho a mi esposo ¿no? A ver, ¿por qué no me ayudas? No me ayudas a aprender Internet, no me ayudas a aprender los mensajes de texto, ¿yo cómo le voy a enseñar a mis hijos, no? ¿Eso es mala onda, no? Y yo me he quedado todo el tiempo abajo, abajo, abajo, fracasada...

\section{Sociopercepción-discriminación-violencia}

La forma en la que la sociedad percibe a la comunidad sorda y en la que la comunidad sorda percibe a la oyente, así como la sociopercepción de otros factores, tales como los roles de género, se relacionan con distintas formas de discriminación.

En este fragmento del grupo focal de la Ciudad de México se aprecia cómo las formas negativas de entender el fenómeno de la sordera a nivel social permean las relaciones familiares de tal manera que generan discriminación y violencia.

Grupo Focal см: 
No sé, por ejemplo, que una mujer, que una mujer oyente se te quede viendo porque eres sorda y entonces eso te molesta: - ¡Ay!, tú eres sorda, tú no sabes, tú eres ignorante. Entonces eso te causa muchísimo enojo y empieza la violencia; por ejemplo, en la familia: - ¡Ay, mira!, mijito tan obediente, tan lindo... ¡Ay! El sordo, que se vaya el sordo, no lo quiero. Entonces eso te va causando violencia; o también, por ejemplo, si estamos haciendo referencia a los hombres sordos: — $¡$ Ay, mira!, yo soy hombre y sé lenguaje de señas muy bien y tú eres mujer y no sabes bien. Tiene que ver como esta lucha de poderes de yo soy mejor, yo sí sé, tú no sabes.

\section{Estrategias de comunicación-autopercepción}

Una de las formas en las que se construye la autopercepción es a partir de las propias capacidades para resolver problemas, lo que está determinado por la comunicación. Las mujeres Sordas reconocen que comunicarse mejor las hace sentir mejor. En el siguiente ejemplo, una integrante del Grupo Focal de sLP reconoce que mientras solo usaba el lenguaje oral con un nivel muy básico se sentía muy poco capaz.

Grupo focal SLP:

yo no sabía cómo defenderme, yo no sabía cómo yo andaba por la vida ahí de borrego, pero hace no mucho que las conocí a todas ellas [participantes del GF]; yo estaba en oralización [ahora se comunica con LSM] y yo hablaba, trataba de hablar con los oyentes; pero, de todas maneras, aunque lo intentaba, este, no podía encajar

Como se muestra en el ejemplo anterior, el manejo de la LSM parece una mejor opción que el limitado acceso al lenguaje oral que, en muchos casos, suele ofrecer la oralización. No obstante, como se evidencia en el siguiente testimonio extraído del Grupo Focal CM, contar con varias estrategias de comunicación tiene efectos aún más benéficos en la autopercepción y la agencia para resolver problemas.

Grupo Focal CM: [respecto de una experiencia en un lugar público]

yo estuve ahí viendo lo que estaba ocurriendo, cómo discutían, cuando el esposo [de una mujer sorda] me ve como que ya no sabía qué hacer y les avisa a los demás. Les decía: ¡cállense, cállense!, nos están viendo. Yo fui y les dije - Tú, ustedes tres [el esposo y otras dos personas] contra un sordo [mujer], no, no, no. A ver, a ver conmigo, a ver conmigo, yo también entiendo lenguaje oral, yo les leo los labios, 
ella no les entiende, si ustedes dos [el esposo y la mujer sorda] tienen un problema, ustedes dos enfréntenlo...

\section{Abuso de sustancias-violencia}

Un factor de riesgo común para la violencia es el abuso de sustancias. En este sentido, las mujeres Sordas no son la excepción. En los testimonios encontramos referencia a este fenómeno, como se aprecia en los siguientes ejemplos de dos participantes del grupo focal de San Luis Potosí y una del grupo focal de Zacatecas.

GF, SLPI: "Mi papá tomaba todos los días, todos los días, le decíamos que no, que no. Y no le importaba, no. Le valía. Entonces se ponía agresiva la cosa, mi hermano me defendía, era lo que hacía".

GF, SLP2: "hace mucho tiempo mi papá tomaba, era una situación tortuosa, muy violenta y varias veces llegaba y nos torturaba a todos. Me jaloneaba y me decía ven, y yo ¿para qué?, ¿para qué?; y a mí no me gustaba cuando estaba así y yo lo que hacía era irme".

GF, ZAC, vía la intérprete:"Era muy borracho [un hombre que conoció], tomador, la dejó embarazada, no le gusta acordarse... Era oyente... era una persona borracha y fue violada y ella no sabía, hasta después ya se dio cuenta de que estaba embarazada, por eso dice que le da vergüenza".

El siguiente diagrama (figura I) es el resultado de un proceso iterativo y, al mismo tiempo, una síntesis de los circuitos identificados que busca representar las relaciones entre las categorías considerando la pregunta analítica ¿Cómo se presenta la violencia de género en mujeres Sordas? En el diagrama se representa la interacción entre las categorías madre y el fenómeno estudiado, la violencia, que está entrelazado con la discriminación-sobreprotección. En la parte superior se ubican los factores protectores: apoyo social y estrategias de comunicación, que atenúan o previenen la violencia, e influyen en forma positiva en la autopercepción de las mujeres. Los factores de riesgo: abuso de sustancias, relaciones familiares negativas y socio-percepción conducen en forma directa a la violencia y con frecuencia también a la discriminación y sobreprotección que afectan negativamente la autopercepción de las mujeres Sordas. En los trayectos entre las categorías se marca el número del circuito $\left(\mathrm{C}_{1}, \mathrm{C}_{2}\right.$, etc.) seguido del número de informantes (grupales o individuales) en el que se identificó. 


\section{Figura I.}

Factores de riesgo y factores protectores de la violencia en una muestra de mujeres Sordas en México

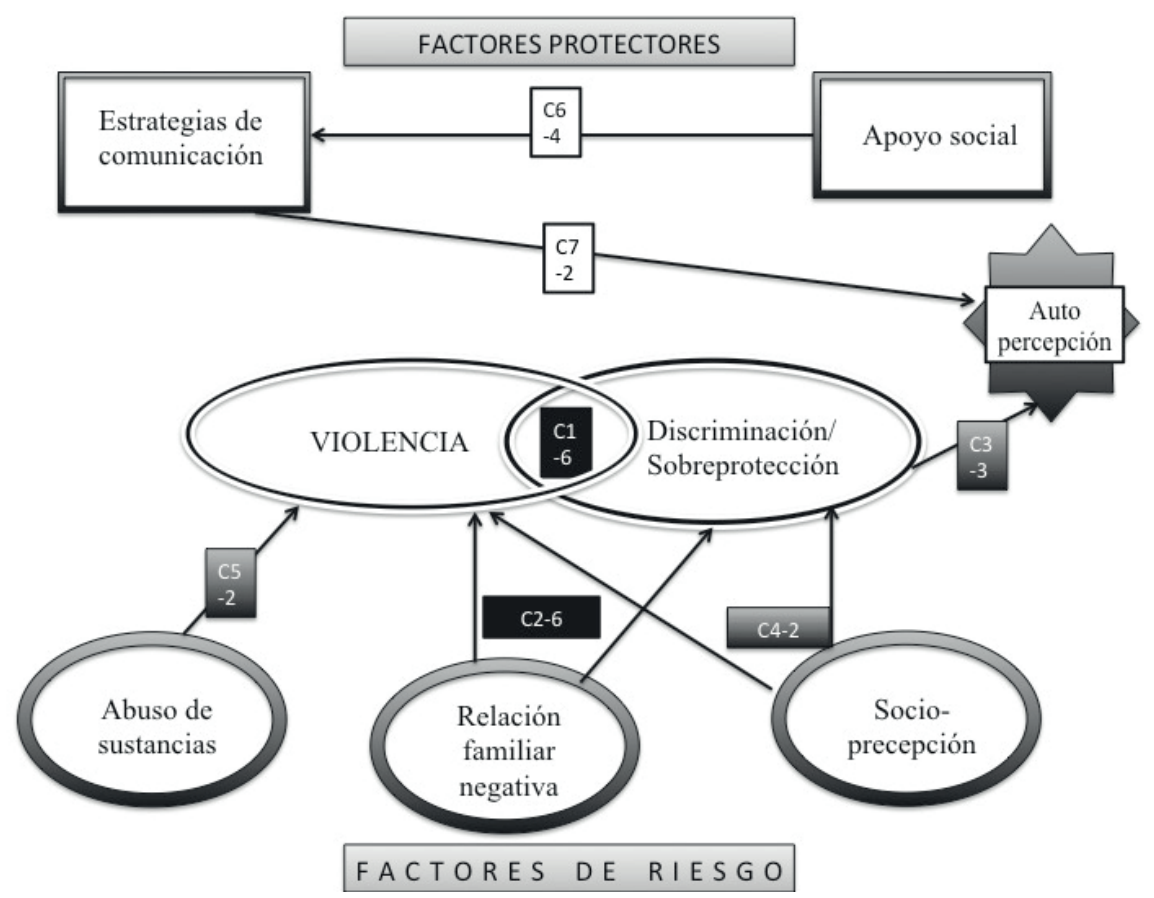

Este trabajo pone de manifiesto, como se revela en el diagrama, que las mujeres Sordas están expuestas a riesgos de violencia desde su nacimiento y dentro de su casa. Sus familias, regularmente conformadas por personas oyentes, suelen carecer de recursos comunicativos (la mayoría no aprende Lengua de Señas, solo lenguajes manuales "caseros"), psicoeducativos y psicosociales con respecto a la sordera para relacionarse adecuadamente con ellas y las violentan o las discriminan. Las familias, por su parte, están inmersas en una sociedad que regularmente no responde de manera empática a la discapacidad en general y a la sordera en particular. La socioprecepción hacia las mujeres Sordas alimenta y refuerza las predisposiciones de la familia. Las mujeres Sordas reciben su carga, muy probablemente con mayor intensidad, de los fenómenos generalizados en la sociedad como violencia intra-familiar y violencia de género, que se asocian al abuso de sustancias. Si bien esto no tendría por qué sorprendernos, sí nos debe alertar. En el trabajo también se identifican los factores protectores: apoyo social y diversidad en las estrategias de 
comunicación. Las instituciones gubernamentales, no gubernamentales y el sistema educativo juegan un papel decisivo en la prevención de la violencia y la discriminación hacia las mujeres Sordas. Los testimonios de las participantes ofrecen evidencia de que cuando hay apoyos adecuados y cuando ellas logran, a través del esfuerzo compartido con familiares o agentes extra familiares, contar con varios recursos comunicativos, la vida les cambia. Cambia primeramente la visión de sí mismas, su autopercepción, lo que las hace conscientes de sus derechos, sus posibilidades de desarrollo, e incluso su potencial para apoyar a otras mujeres Sordas.

\section{Discusión}

El objetivo de nuestro estudio fue el de conocer la percepción de la violencia de género en mujeres Sordas y describir la visión global de los factores que intervienen en la violencia de género hacia las mujeres Sordas en México. La figura I resume esta visión, la cual se desprende del análisis cualitativo del discurso de las participantes, ya sea a través de entrevistas o de grupos focales.

En este sentido, las mujeres Sordas que participaron en este estudio destacan el papel del apoyo social y las estrategias de comunicación como factores protectores, como ha sido descrito en la literatura reciente (Morales-Acosta y Aguilar-Caro, 2018). El uso de la lengua de señas con otras personas Sordas y el apoyo derivado de quien comparte una misma lengua y una cultura reducen la posibilidad de sufrir violencia y aumentan las posibilidades de construir un autoconcepto y una autoestima positivos.

No obstante, los principales factores que conducen directa o indirectamente a las mujeres Sordas a sufrir violencia son el abuso de sustancias (Anderson, 2010; Anderson y Leigh, 20II), las relaciones familiares negativas y de sobreprotección. Todos estos factores se producen en la familia de las mujeres Sordas o bien en su entorno laboral, educativo o social, por lo que, según nuestras observaciones, el autoconcepto y la autopercepción se ven dañadas en detrimento de su autoestima y les generan una idea de minusvalía e indefensión hacia sí mismas.

Finalmente, la sociopercepción de los roles de género de las mujeres Sordas, tanto desde dentro como desde fuera de la comunidad sorda y la discriminación múltiple (Makkonen, 2002) que percibieron las mujeres de nuestro estudio, constituyeron factores de riesgo que afectan negativamente la autopercepción y conllevan a la violencia, que a su vez refuerza la sociopercepción negativa y la discriminación hacia las mujeres Sordas, y todo ello genera un circuito negativo de exclusión. Recientemente 
el estudio cualitativo de Al-Makhamreh (2016) documentó en población sorda en Jordania resultados similares a los nuestros y coincide en que la escuela y la familia son los entornos predominantes de exclusión social para este grupo de la población.

Como podemos observar, la percepción de la violencia de las mujeres Sordas coincide con la teoría interseccional (Raj, 2002) con la cual analizamos la convergencia de múltiples discriminaciones que contribuyen directa o indirectamente a generar desigualdad social, exclusión social y violencia sistemática. En efecto, la suma de la discriminación reportada por ser mujer, más la propiciada por motivos de tener una discapacidad auditiva, más la generada por las distintas barreras sociales reportadas colocan a las participantes en una situación de enorme desventaja social. En el mismo tenor, la teoría interseccional permite identificar la convergencia de múltiples discriminaciones dentro de la muestra o entre las mismas mujeres Sordas. Las tasas de mayor o menor exclusión social y violencia sistemática reportada entre las mujeres Sordas con respecto a sus homólogas oyentes podrían depender de factores tales como: estado civil, nivel educativo, situación familiar, laboral y geolocalización, tales como: al ser madre soltera con escolaridad básica con la que solo se pueden obtener trabajos pobremente remunerados y/o con ocupación sin remuneración y/o con baja compatibilidad con la pareja por tratarse de un hombre oyente. Resulta evidente que, aunque las mujeres sordas pueden diferenciarse en cuanto a sus posiciones sociales, el tipo de control social ejercido sobre ellas y de su agencia en el ámbito del complejo sistema social en que se incluyen, la mayoría presentan varios niveles de vulnerabilidad que las predispone a ser víctimas de violencia de género (Couto et al., 2019).

La situación de vulnerabilidad de las mujeres sordas se agudiza por la falta de políticas públicas con perspectiva de discapacidad y género (De la Rosa, 2013). En otras palabras, la falta de acceso a servicios públicos en general atenta contra los derechos humanos de la población sorda (Estrada, 2008), y más particularmente contra su derecho a la salud mental, pues al no poder comunicarse para gestionar servicios básicos de salud, educación, protección legal, etc. y dada la falta de profesionales que usen la lengua de señas mexicana para comunicarse o de intérpretes efectivos, se genera incomunicación, desconfianza y estrés e incluso conflictos mayores entre los presentadores de servicios y los usuarios sordos.

Los resultados de este estudio coinciden con los reportados de España sobre la situación de las mujeres Sordas ante la violencia de género (CNSE, 2009a). No obstante, la situación mexicana es aún más grave, pues la realidad en México para las personas Sordas es de mayor desventaja. Por una parte, las leyes y reglamentaciones españolas tienen un enfoque más claramente inclusivo; por la otra, la sociedad 
Sorda española se encuentra más organizada que la mexicana en asociaciones que promueven la inclusión y los derechos humanos.

Realizar investigación con la población Sorda presenta diversos desafíos. A lo largo de este trabajo identificamos varias limitaciones que logramos sortear y otras que nos fue imposible remediar y de las que damos cuenta para ser consideradas en la valoración del trabajo, así como para futuras investigaciones:

La persona con hipoacúsia que colaboró en las cuatro primeras entrevistas en San Luis Potosí, a pesar de ser reconocida por la comunidad como intérprete, no logró hacer una buena traducción e interpretación del material, lo que causó que se perdiera alguna información. Ella hablaba (traducía) en tercera persona (habla reportada) y realizaban preguntas desde su criterio sin tomar en cuenta la guía o al entrevistador. Habría sido deseable contar con intérpretes de LSM certificados, no solo reconocidos; sin embargo, las personas con ese perfil son muy escasas en nuestro país y particularmente en SLP, pues, aunque no se cuenta con información sobre los intérpretes de LSM en los estados, sí se sabe que de los 40 certificados en el país, la cuarta parte radica en la Ciudad de México (Godoy, 2015).

En la recolección de datos, si bien tuvimos especial cuidado en contar con equipo semi-profesional, los mismos investigadores y algunos voluntarios realizamos el montaje para las filmaciones. Esto ocasionó que en algunas videograbaciones la cámara no enfocara apropiadamente a los participantes y se perdía su lenguaje corporal. Con base en esta experiencia y a pesar del costo económico que representa, consideramos que en trabajos con LSM es indispensable contar con apoyo técnico profesional.

Nuestro grupo de trabajo está formado por un equipo interdisciplinario de investigadores consolidados e investigadores en formación; no obstante, ninguno forma parte de la comunidad Sorda, lo que en ocasiones limitó el acceso a informantes y otros aspectos del proceso. Nuestro abordaje seguramente habría sido más acertado si dentro del equipo hubiéramos contado con algún participante Sordo. La participación de la comunidad Sorda en estudios de este tipo es de gran relevancia para alcanzar una mayor "solidez cultural". En estudios futuros similares consideramos que se debe incorporar a una mujer Sorda al equipo de investigadores.

Para futuros estudios cualitativos con la comunidad Sorda en México se recomienda contar con intérpretes certificados en LSM, así como apoyo técnico profesional para asegurar una adecuada recolección e interpretación de datos cualitativos. Por último, pero no menos importante, el conocimiento generado por este tipo de estudio será de una mayor relevancia cultural si personas de la comunidad Sorda participan en todas las etapas de investigación. 


\section{Referencias}

Al-Makhamreh, Sahar

2016 "Hearing the voices of young deaf people: Implications for social work practice in Jordan", International Social Work, 59(I), pp. 47-59, doi: IO.1177/0020872813499057

Anderson, Melissa Lee

2010 Prevalence and predictors of intimate partner violence victimization in the deaf community, Gallaudet University.

Anderson, Melissa Lee e Irene W. Leigh

20 II "Intimate partner violence against deaf female college students", Violence Against Women, I7(7), pp. 822-834, doi: 10.1177/I077801211412544

Bloor, Michael, Jane Frankland, Michelle Thomas y Kate Robson (eds.) 200I Focus groups in social research, SAGE Publications.

Cámara de Diputados del H. Congreso de la Unión

2015 Ley General de Acceso de las Mujeres a una Vida Libre de Violencia. https://www.gob.mx/cms/uploads/attachment/file/209278/Ley_ General_de_Acceso_de_las_Mujeres_a_una_Vida_Libre_de_Violencia.pdf

Casique, Irene y Roberto Castro

2012 "Retratos de la violencia contra las mujeres en México: Análisis de resultados de la Encuesta Nacional sobre la Dinámica de las Relaciones en los Hogares 20II", Cuadernos de Trabajo 35, Instituto Nacional de la Mujeres/Centro Regional de Investigaciones Multidisciplinarias-UNAM.

Confederación Estatal de Personas Sordas [CNSE]

2009a Situación de las mujeres sordas ante la violencia de género, Madrid, Confederación Estatal de Personas Sordas.

Confederación Estatal de Personas Sordas [CNSE]

2009b Mujeres sordas ante la violencia de género. Informe con propuestas de accesibilidad, Madrid, Confederación Estatal de Personas Sordas.

Couto, Marcia Teresa; Elda de Oliveira, Marco Antonio Separavich Alves y Olinda do Carmo Luiz

2019 "La perspectiva feminista de la interseccionalidad en el campo de la salud pública: revisión narrativa de las producciones teórico-metodológicas", Salud Colectiva, 15e, pp. I-I4, doi: 10.18294/sc.2019.1994 
De la Rosa Prieto, Alejandra

2013 "Discriminación múltiple: mujeres con discapacidad en México", Género y Salud en cifras, II(2), pp. 2I-30.

Diario Oficial de la Federación [Dof]

2009 Acuerdo so/I-09/o2-S http://dof.gob.mx/nota_detalle_popup. php? $\operatorname{codigo}=5093812$

Estrada Aranda, Benito

2008 "La vulneración de los derechos humanos de las personas Sordas en México", Derechos Humanos México. Revista del Centro Nacional de Derechos Humanos, 3(8), pp. I05-I27.

Frías, Sonia, $\mathrm{M}$

2017 " 25 años de investigación cuantitativa y cualitativa sobre violencia en contra de las mujeres en México", Realidad, Datos y Espacio: Revista Internacional de Estadistica y Geografía, 8(2), pp. 5-57.

Godoy, Emilio

2015 Los intérpretes de lengua de señas mexicana intentan afrontar la discriminación por discapacidad, Equal Times (www.equaltimes.org), https:// www.equaltimes.org/los-interpretes-de-lengua-de-senas\#.YH4X_ kjolQm [consulta: 13/03/2015].

Ibarra, Lorena y Nancy Muñoz Espinosa

201 "Abuse, violence and deaf women", en: Benito Estrada Aranda (ed.), Mental Health and some sociocultural issues in deaf people, Nueva York, Nova Publisher, pp. 2I-50.

Instituto Nacional de las Mujeres [INM]

2002 Mujeres y discapacidad en México, México, Instituto Nacional de las Mujeres, http://cedoc.inmujeres.gob.mx/documentos_download/roo860.pdf

Instituto Nacional de Estadística y Geografía [INEGI]

2004 Encuesta Nacional sobre la Dinámica de las Relaciones en los Hogares 2003, ENDIREH, México, INEGI.

Instituto Nacional de Estadística y Geografía [INEGI]

2007 Encuesta Nacional sobre la Dinámica de las Relaciones en los Hogares 2006, eNDIREH, México, INEGI.

Instituto Nacional de Estadística y Geografía [INEGI]

2016 Encuesta Nacional sobre la Dinámica de las Relaciones en los Hogares 2016, ENDIREH, México, INEGI 
Instituto Nacional de Estadística y Geografía [INEGI]

2010 Xirl Censo general de población y vivienda 2010.

Instituto Nacional de Salud Pública [INSP]

2003 Encuesta Nacional sobre Violencia contra las Mujeres, México, INSP.

López González, María

2007 "Discapacidad y Género: estudio etnográfico sobre mujeres discapacitadas", Educación y diversidad = Education and diversity: Revista inter-universitaria de investigación sobre discapacidad e interculturalidad, I, pp. 137-I72

Makkonen, Timo

2002 Multiple, compound and intersectional discrimination: Bringing the experiences of the most marginalized to the fore, Institute for Human Rights, Åbo Akademi University.

Mason, Teresa Crowe

2010 "Does knowledge of dating violence keep deaf college students at Gallaudet University out of abusive relationships?", Journal of the American Deafness E Rehabilitation Association, 43(2), pp. 74-9I.

McCarthy Katherine, J.; Mehta, Ruchi; \& Haberland, Nichole A 2018 "Gender, power, and violence: A systematic review of measures and their association with male perpetration of IPV", PLOS ONE, I3(II), pp. I-27, doi: 10.137I/journal.pone.020709I

Mitchell, Ross E. y Michael A. Karchmer

2004 "Chasing the mythical ten percent: Parental hearing status of deaf and hard of hearing students in the United States", Sign Language Studies, 4(2), pp. 138-163, doi: 10.1353/sls.2004.0005

Minguet Soto, Amparo

2000 Rasgos sociológicos y culturales de las personas sordas, Valencia, Fundación FESORD C.V [Federación de Sordos de la Comunidad de Valencia]

Ministerio de Sanidad, Servicios Sociales e Igualdad [MSssi]

2012 Macroencuesta de Violencia de Género 2011: principales resultados, https://igualdad.uniovi.es/c/document_library/get_file?uui$\mathrm{d}=5 \mathrm{~b} 422918$-di43-44ae-b880-Id 3858 bfeffc $\&$ groupId $=336079$

Ministerio de Sanidad, Servicios Sociales e Igualdad [MSssi] 2015 Macroencuesta de Violencia contra la mujer 2015, https://violenciagenero,igualdad.gob.es/violenciaEnCifras/estudios/colecciones/pdf/ Libro_22_Macroencuesta2015.pdf 
Morales-Acosta, Gina V. y Aura I. Aguilar-Caro

2018 "Diversidad sorda y violencia de género: restricción comunicativa en el uso de la lengua de señas en salud", fem: Revista de la Fundación Educación Médica, 2I(6), pp. 309-313.

Morgan, David. L.

1997 Focus groups as qualitative research, SAGE Publications, doi: I0.4135/978I4I2984287

Muñoz Bravo, Javier

2007 "Sordera y Salud mental: la psicología frente a la deficiencia auditiva", Infocoponline Revista de Psicología, http://www.infocop.es/view_article. asp? cat $=57 \& \mathrm{id}=879$

Obinna, Jennifer; Sarah Krueger, Constance Osterbaan, Jane M. Sadusky y Wendy deVore

2006 Understanding the needs of the victims of sexual assault in the deaf community. Final report submitted to the National Institute of Justice, Washington, DC. (NCJ 212867), www.ncjrs.gov/pdffilesi/nij/grants/212867. pdf

Pino López, Felisa R

2007 La cultura de las personas sordas, https://epclaudiagv.files.wordpress. com/20I4/II/laculturadelaspersonassordasfelisar.pdf

Raj, Rita

2002 Women at the intersection: Indivisible rights, identities, and oppressions, EUA, Centre for Women's Global Leadership, Rutgers, the State University of New Jersey.

Red Internacional de Mujeres con Discapacidad [International Network of Women with Disability, INwwD]

2010 Violencia contra Mujeres con Discapacidad, https://inwwd.files.wordpress.com/2010/II/final2oroinwwd-violenciacontramujerescondiscapacidad_esp.doc

Rems-Smario, Julie

2007 "Domestic violence: We can't ignore it anymore", NADmag, marzo-abril, pp. I6-I8, https://issuu.com/nadi88o/docs/2007_vol7_no2

SIPSE.COM

2016 En México se hacen ciegos ante los sordos, https://sipse.com/mexico/ sordos-discapacidad-gobierno-mexico-224324.html 
Shum, Grace Mun Man; Ángeles Conde Rodríguez e Inés Portillo Mallorca 2006 Mujer, discapacidad y violencia. El rostro oculto de la desigualdad, Madrid, Ministerio de Trabajo y Asuntos Sociales.

Strauss, Anselm L. y Juliet Corbin

2002 Bases de la investigación cualitativa: técnicas y procedimientos para desarrollar la teoría fundamentada, Medellín, Universidad de Antioquia.

Waston, Cherire

2014 Violence in deaf cultureः My story, my voice. Dissenting Voices, 3(I), pp. 8I-98, http://digitalcommons.brockport.edu/dissentingvoices/vol3/ issi $/ 9$

Wojcik, Michelle L.T., Batya Y. Rubenstein, Amber. A. Petkus, Maria Racadio, Valerie R. Anderson, Bonnie S. Fisher, Pamela Wilcox y Amy Bleser

202I "Coming Together in the Fight Against Intimate Partner Violence: Lessons Learned From a Researcher-Practitioner Collaboration Evaluating Cincinnati's Domestic Violence Enhanced Response Team (DVERT)", Journal of Contemporary Criminal Justice, 37(2), pp. 22I-243, doi. IO.II77/IO4398622199986I

Wright, Kevin, Jillian J. Turanovic, Eryn N. O’Neal, Stephanie J. Morse y Evan T. Booth

2016

"The cycle of violence revisited: Childhood victimization, resilience, and future violence", Journal of Interpersonal Violence. 34, pp. I-26 doi: I0.1177/0886260516651090

Benito Daniel Estrada Aranda

Profesor-investigador adscrito a la Facultad de Psicología de la Universidad Autónoma de San Luis Potosí. Cuenta con un doctorado en Psicología y maestría en Terapia Sistémica. Ejerce como psicoterapeuta en práctica privada. Es miembro del Sistema Nacional de Investigadores desde 2007, Nivel I. Participa como miembro del núcleo básico y titular de la coordinación académica en el Doctorado en Psicología de la Universidad Autónoma de San Luis Potosí (UASLP). Además, es líder del Cuerpo Académico en Psicología de la Salud y Psicoterapia. Sus principales líneas de investigación son psicología de la salud, salud mental y sordera, y psicoterapia. 
Silvia Romero Contreras

Profesora investigadora de la Universidad Autónoma de San Luis Potosí adscrita en la Facultad de Psicología. Cuenta con doctorado en Educación por la Universidad de Harvard y con la distinción de Investigadora Nacional Nivel iI (Sistema Nacional de Investigadores del Consejo Nacional de Ciencia y Tecnología). Fue receptora de la beca Fullbright-García Robles. Participa como miembro del núcleo básico del Posgrado Multidisciplinario en Ciencias Ambientales y el Doctorado en Psicología de la UASLP. Es líder del Cuerpo Académico consolidado UASLP-I96: Evaluación e intervención en psicología y educación. Sus principales líneas de investigación son educación especial e inclusiva, formación docente y educación comunitaria-inclusiva, temáticas que aborda con métodos mixtos, cuantitativo-cualitativos.

Carmen Delgado Álvarez

Catedrática de la Universidad Pontificia de Salamanca (UPSA), licenciada y doctora en Psicología en el área de Metodología de la Investigación, y profesora titular de Psicometría y Estadística Multivariante, así como directora del posgrado de Intervención Multidisciplinar en Violencia de Género, disciplina en la que está reconocida como una de las grandes especialistas e investigadoras de España. Decana de la Facultad de Psicología de la Universidad Pontificia de Salamanca en 2009, y Secretaria General de dicha universidad.

Nicholas Timothy Kaufmann

Profesor-investigador de la Universidad Autónoma de San Luis Potosí, adscrito a la Facultad de Psicología. Es candidato al Sistema Nacional de Investigadores y miembro del núcleo básico de la Maestría en Derechos Humanos de la UASLP. Tiene un doctorado en Psicología Social-Comunitaria por la Universidad de Illinois en Chicago. Pertenece al Cuerpo Académico consolidado UASLP-196: Evaluación e intervención en psicología y educación. Sus principales líneas de investigación son: diseño, implementación y evaluación de intervenciones preventivo-promocionales en salud integral con perspectiva de género. 
Citar como: Benito Estrada Aranda, Silvia Romero Contreras, Carmen Delgado Álvarez, Nicholas T. Kaufmann (2022), "Percepción de la violencia de género de las mujeres sordas mexicanas”, Iztapalapa. Revista de Ciencias Sociales y Humanidades, núm. 92, año 43, enero-junio de 2022, ISSN: 20079176; pp. I89-220. Disponible en <http://revistaiztapalapa.izt.uam.mx/ index.php/izt/issue/archive $>$. 